\title{
Three-family left-right symmetry with low-scale seesaw mechanism
}

\section{Mario Reig, José W.F. Valle and C.A. Vaquera-Araujo}

AHEP Group, Institut de Física Corpuscular - C.S.I.C., Universitat de València, Parc Científic de Paterna, C/ Catedrático José Beltrán, 2 E-46980 Paterna (Valencia), Spain

E-mail: mareiglo@alumni.uv.es, valle@ific.uv.es, vaquera@ific.uv.es

ABSTRACT: We suggest a new left-right symmetric model implementing a low-scale seesaw mechanism in which quantum consistency requires three families of fermions. The symmetry breaking route to the Standard Model determines the profile of the "next" expected new physics, characterized either by the simplest left-right gauge symmetry or by the 3-3-1 scenario. The resulting $Z^{\prime}$ gauge bosons can be probed at the LHC and provide a production portal for the right-handed neutrinos. On the other hand, its flavor changing interactions would affect the K, D and B neutral meson systems.

Keywords: Beyond Standard Model, Gauge Symmetry, Neutrino Physics

ArXiv EPrint: 1611.04571 


\section{Contents}

1 Introduction 1

2 The model 2

3 Spontaneous symmetry breaking 3

4 Phenomenological implications 5

$5 \quad$ Flavor-changing neutral currents $\quad 6$

$\begin{array}{llr}6 & \text { Conclusion } & 7\end{array}$

\section{Introduction}

Both the great success as well as the shortcomings of the Standard Model (SM) in providing a full picture of reality are well publicized. Amongst the latter are the facts that it fails in giving a convincing explanation for small neutrino masses, the existence three families, and the dynamical origin of parity violation. Taking these as clues, we suggest a new Standard Model extension which provides a dynamical criterion for selecting the profile characterizing the "next step" towards physics Beyond the Standard Model. This would provide an answer to a current burning question, after the lack of convincing new signals in Run 2 of the LHC: what is the expected pattern of the upcoming new physics? By suggesting that these three features are deeply inter-connected we reconcile the idea of spontaneous parity violation with anomaly cancellation with, and only with, three families of fermions.

The framework is based on a generalized left-right symmetric $\mathrm{SU}(3)_{\mathrm{c}} \otimes \mathrm{SU}(3)_{\mathrm{L}} \otimes$ $\mathrm{SU}(3)_{\mathrm{R}} \otimes \mathrm{U}(1)_{\mathrm{X}}$ group structure, first proposed in [1] in a different context. In [2] we suggested a unique framework able to mimic either a usual left-right symmetric theory or a 331 model, incorporating spontaneous parity violation and small neutrino masses through the seesaw mechanism. In order to incorporate the cancellation of the Adler-Bell-Jackiw anomalies $[3,4]$ the number of fermion families must chosen to match exactly the number of colors, with quarks assigned in a non-sequential way, in which one quark family differs from the other two [5], as in the early proposal of refs. [5, 6] (see [7] for an alternative formulation). ${ }^{1}$ We choose the simplest scalar sector for which light neutrino masses are proportional to a small parameter, implementing a genuine low-scale realization of the seesaw mechanism, such as the inverse [10-15] or linear seesaw mechanism [12-14], instead

\footnotetext{
${ }^{1}$ The model is unique up to exotic fermion charge assignments. Unfortunately, the interesting choice made in $[8,9]$ is inconsistent with our construction, due to the perturbativity requirement of the gauge couplings $[1]$.
} 


\begin{tabular}{|c|c|c|c|c|c|c|c|c|c|c|c|}
\hline & $\psi_{L}^{a}$ & $\psi_{R}^{a}$ & $Q_{L}^{\alpha}$ & $Q_{R}^{\alpha}$ & $Q_{L}^{3}$ & $Q_{R}^{3}$ & $S_{L}^{a}$ & $\Phi$ & $\rho$ & $\chi_{L}$ & $\chi_{R}$ \\
\hline $\mathrm{SU}(3)_{\mathrm{c}}$ & $\mathbf{1}$ & $\mathbf{1}$ & $\mathbf{3}$ & $\mathbf{3}$ & $\mathbf{3}$ & $\mathbf{3}$ & $\mathbf{1}$ & $\mathbf{1}$ & $\mathbf{1}$ & $\mathbf{1}$ & $\mathbf{1}$ \\
\hline $\mathrm{SU}(3)_{\mathrm{L}}$ & $\mathbf{3}$ & $\mathbf{1}$ & $\mathbf{3}^{*}$ & $\mathbf{1}$ & $\mathbf{3}$ & $\mathbf{1}$ & $\mathbf{1}$ & $\mathbf{3}$ & $\mathbf{3}$ & $\mathbf{3}$ & $\mathbf{1}$ \\
\hline $\mathrm{SU}(3)_{\mathrm{R}}$ & $\mathbf{1}$ & $\mathbf{3}$ & $\mathbf{1}$ & $\mathbf{3}^{*}$ & $\mathbf{1}$ & $\mathbf{3}$ & $\mathbf{1}$ & $\mathbf{3}^{*}$ & $\mathbf{3}$ & $\mathbf{1}$ & $\mathbf{3}$ \\
\hline $\mathrm{U}(1)_{\mathrm{X}}$ & $-\frac{1}{3}$ & $-\frac{1}{3}$ & 0 & 0 & $\frac{1}{3}$ & $\frac{1}{3}$ & 0 & 0 & $\frac{1}{3}$ & $-\frac{1}{3}$ & $-\frac{1}{3}$ \\
\hline
\end{tabular}

Table 1. Particle content of the model, with $a=1,2,3$ and $\alpha=1,2$.

of the more conventional high-scale seesaw [16-20]. Moreover the model suggests that the CKM matrix characterizing quark weak interactions may have a dynamical origin through a new "leptophobic" vacuum expectation value. The scheme has potentially accessible new neutral gauge bosons coupling non-diagonally to the quark flavors, inducing flavor changing neutral currents in the K, D and B neutral meson systems [21]. On the other hand such $Z$ 's can also be searched directly in Dilepton studies at the LHC [22]

This paper is organized as follows. First we sketch our framework reconciling the existence of parity at a fundamental level with a gauge structure consistent only for the case of three families of fermions. We then show how different patterns of symmetry breaking determine alternative profiles for the "next" expected physics beyond the Standard Model. Finally we briefly discuss generic features of fermion masses and mixings, as well as their interactions and phenomenological implications for the LHC and $\mathrm{K}, \mathrm{D}$ and B meson mixing.

\section{The model}

The gauge group is $\mathrm{SU}(3)_{\mathrm{c}} \otimes \mathrm{SU}(3)_{\mathrm{L}} \otimes \mathrm{SU}(3)_{\mathrm{R}} \otimes \mathrm{U}(1)_{\mathrm{X}}$ with the three families of fermion fields assigned as,

$$
\psi_{L, R}^{a}=\left(\begin{array}{c}
\nu \\
\ell^{-} \\
N
\end{array}\right)_{L, R}^{a}, \quad Q_{L, R}^{\alpha}=\left(\begin{array}{c}
d \\
-u \\
D
\end{array}\right)_{L, R}^{\alpha}, \quad Q_{L, R}^{3}=\left(\begin{array}{c}
u \\
d \\
U
\end{array}\right)_{L, R}^{3}
$$

where parity acts as: $\psi_{L}^{a} \leftrightarrow \psi_{R}^{a}, Q_{L}^{\alpha} \leftrightarrow Q_{R}^{\alpha}, Q_{L}^{3} \leftrightarrow Q_{R}^{3}$.

The particle content of the model is summarized in table 1 . We also introduce the fermion fields $S_{L}^{a}$, transforming as singlets under the $\mathrm{SU}(3)_{\mathrm{c}} \otimes \mathrm{SU}(3)_{\mathrm{L}} \otimes \mathrm{SU}(3)_{\mathrm{R}} \otimes \mathrm{U}(1)_{\mathrm{X}}$ gauge symmetry and crucial ingredients of low-scale seesaw schemes [10] as well as a set of scalar multiplets including a bi-triplet scalar $\Phi$ involved in the generation of charged fermion masses, as well as $\chi_{L}$ and $\chi_{R}$ triggering neutrino masses, see below. Notice that, following the procedure used in refs. [12-14], in the current model we replace the sextets $\Delta_{L}$ and $\Delta_{R}$ present in the construction of refs. [2, 23], by the fields $\chi_{L}$ and $\chi_{R}$

$$
\chi_{L} \sim\left(1,3,1,-\frac{1}{3}\right), \quad \chi_{R} \sim\left(1,1,3,-\frac{1}{3}\right)
$$


In the presence of the gauge singlet fermions, the Yukawa interactions for leptons are given by

$$
-\mathcal{L}_{\text {leptons }}=\sum_{a, b}\left[y_{R}^{a b} \bar{\psi}_{R}^{a} \chi_{R} S_{L}^{b}+y_{L}^{a b} \bar{\psi}_{L}^{a} \chi_{L}\left(S_{L}^{b}\right)^{c}+y^{a b} \bar{\psi}_{L}^{a} \Phi \psi_{R}^{b}+\frac{\mu_{a b}}{2} \overline{\left(S_{L}^{a}\right)^{c}} S_{L}^{b}\right]+\text { h.c. }
$$

Parity acts on singlet fermions as $S_{L} \leftrightarrow\left(S_{L}\right)^{c}$ and the scalar fields transform as $\Phi \leftrightarrow \Phi^{\dagger}$, $\chi_{L} \leftrightarrow \chi_{R}$, which implies that $y_{R}=y_{L}$ and $y=y^{\dagger}$. Spontaneous symmetry breaking is driven by the following structure of vacuum expectation values (VEVs): ${ }^{2}$

$$
\begin{array}{cc}
\langle\Phi\rangle=\frac{1}{\sqrt{2}} \operatorname{diag}\left(k_{1}, k_{2}, n\right), & \langle\rho\rangle=\frac{1}{\sqrt{2}}\left(\begin{array}{ccc}
0 & k_{3} & 0 \\
k_{4} & 0 & 0 \\
0 & 0 & 0
\end{array}\right), \\
\left\langle\chi_{R}\right\rangle=\frac{1}{\sqrt{2}}\left(\begin{array}{c}
v_{R} \\
0 \\
\Lambda_{R}
\end{array}\right), \quad\left\langle\chi_{L}\right\rangle=\frac{1}{\sqrt{2}}\left(\begin{array}{c}
v_{L} \\
0 \\
\Lambda_{L}
\end{array}\right) .
\end{array}
$$

In this work, we assume for simplicity $k_{4}, \Lambda_{L}=0$ and the hierarchy $n, v_{R}, \Lambda_{R} \gg k_{i}, v_{L}$, $i=1, \ldots, 3$. After symmetry breaking the electric charge is identified as the unbroken combination

$$
Q=T_{L}^{3}+T_{R}^{3}+\beta\left(T_{L}^{8}+T_{R}^{8}\right)+X,
$$

and the parameter $\beta$ is given, in our construction, by

$$
\beta=-1 / \sqrt{3},
$$

which corresponds to the SVS case [5].

\section{Spontaneous symmetry breaking}

The most general scalar potential characterizing symmetry breaking in our model can be written as

$$
\begin{aligned}
V= & V_{\chi}+V_{\Phi}+V_{\rho}+V_{\Phi \chi}+V_{\Phi \rho}+V_{\chi \rho}+V_{\Phi \rho \chi}, \\
V_{\chi}= & \mu_{\chi}^{2}\left(\chi_{L}^{\dagger} \chi_{L}+\chi_{R}^{\dagger} \chi_{R}\right)+\lambda_{1}\left[\left(\chi_{L}^{\dagger} \chi_{L}\right)^{2}+\left(\chi_{R}^{\dagger} \chi_{R}\right)^{2}\right]+\lambda_{2} \chi_{L}^{\dagger} \chi_{L} \chi_{R}^{\dagger} \chi_{R}, \\
V_{\Phi}= & \mu_{\Phi}^{2} \operatorname{Tr}\left(\Phi \Phi^{\dagger}\right)+f_{\Phi}\left[\epsilon^{l_{1} l_{2} l_{3}} \epsilon_{r_{1} r_{2} r_{3}} \Phi_{l_{1}}^{r_{1}} \Phi_{l_{2}}^{r_{2}} \Phi_{l_{3}}^{r_{3}}+\text { h.c. }\right]+\lambda_{3} \operatorname{Tr}\left(\Phi \Phi^{\dagger}\right)^{2}+\lambda_{4} \operatorname{Tr}\left(\Phi \Phi^{\dagger} \Phi \Phi^{\dagger}\right), \\
V_{\rho}= & \mu_{\rho}^{2} \operatorname{Tr}\left(\rho \rho^{\dagger}\right)+\lambda_{5} \operatorname{Tr}\left(\rho \rho^{\dagger}\right)^{2}+\lambda_{6} \operatorname{Tr}\left(\rho \rho^{\dagger} \rho \rho^{\dagger}\right), \\
V_{\Phi \chi}= & f_{\chi}\left(\chi_{L}^{\dagger} \Phi \chi_{R}+\text { h.c. }\right)+\lambda_{7}\left(\chi_{L}^{\dagger} \chi_{L}+\chi_{R}^{\dagger} \chi_{R}\right) \operatorname{Tr}\left(\Phi \Phi^{\dagger}\right)+\lambda_{8}\left(\chi_{L}^{\dagger} \Phi \Phi^{\dagger} \chi_{L}+\chi_{R}^{\dagger} \Phi^{\dagger} \Phi \chi_{R}\right), \\
V_{\Phi \rho}= & \lambda_{9} \operatorname{Tr}\left(\rho \rho^{\dagger}\right) \operatorname{Tr}\left(\Phi \Phi^{\dagger}\right)+\lambda_{10}\left[\operatorname{Tr}\left(\Phi \Phi^{\dagger} \rho \rho^{\dagger}\right)+\operatorname{Tr}\left(\Phi^{\dagger} \Phi \rho^{T} \rho^{*}\right)\right] \\
& +\lambda_{11}\left[\operatorname{Tr}\left(\Phi^{\dagger} \rho \Phi^{T} \rho^{*}\right)+\operatorname{Tr}\left(\Phi \rho^{T} \Phi^{*} \rho^{\dagger}\right)\right] \\
V_{\chi \rho}= & \lambda_{12} \operatorname{Tr}\left(\rho \rho^{\dagger}\right)\left(\chi_{L}^{\dagger} \chi_{L}+\chi_{R}^{\dagger} \chi_{R}\right)+\lambda_{13}\left(\chi_{L}^{\dagger} \rho \rho^{\dagger} \chi_{L}+\chi_{R}^{\dagger} \rho^{T} \rho^{*} \chi_{R}\right), \\
V_{\Phi \rho \chi}= & f_{\rho}\left[\epsilon^{l_{1} l_{2} l_{3}}\left(\Phi \rho^{T}\right)_{l_{1} l_{2}}\left(\chi_{L}\right)_{l_{3}}+\epsilon_{r_{1} r_{2} r_{3}}\left(\Phi^{\dagger} \rho\right)^{r_{1} r_{2}}\left(\chi_{R}\right)^{r_{3}}+\text { h.c. }\right] \\
& +\lambda_{14}\left(\Phi \rho^{T} \Phi \chi_{R}+\Phi^{\dagger} \rho \Phi^{\dagger} \chi_{L}+\text { h.c. }\right),
\end{aligned}
$$

\footnotetext{
${ }^{2}$ We restrict our analysis to the case in which there is no mixing at tree level between exotic and SM fermions. Note that, for the most general VEV structure a small mixing between Standard Model and exotic fermions is induced, but it will be controlled by the ratio $k_{i} / n$ which is expected to be small.
} 
Minimization leads to the following tadpole equations:

$$
\begin{aligned}
\mu_{\Phi}^{2}= & -\left(n^{2}+k_{1}^{2}+k_{2}^{2}\right) \lambda_{3}-\left(n^{2}+k_{2}^{2}\right) \lambda_{4}-\frac{1}{2}\left[k_{3}^{2} \lambda_{9}-\frac{k_{2}^{2} k_{3}^{2}}{n^{2}-k_{2}^{2}} \lambda_{10}+\frac{n^{2} \Lambda_{R}^{2}}{n^{2}-k_{2}^{2}} \lambda_{8}+\left(v_{L}^{2}+v_{R}^{2}+\Lambda_{R}^{2}\right) \lambda_{7}\right], \\
\mu_{\rho}^{2}= & -\frac{1}{2}\left[\frac{v_{L}^{2} \Lambda_{R}^{2}\left(v_{L}^{2}-v_{R}^{2}-\Lambda_{R}^{2}\right)}{k_{3}^{2}\left(v_{L}^{2}-v_{R}^{2}\right)}\left(2 \lambda_{1}-\lambda_{2}\right)+k_{3}^{2}\left(\lambda_{5}+\lambda_{6}\right)+\frac{\left(n^{2}-k_{1}^{2}\right) \Lambda_{R}^{2}}{k_{3}^{2}} \lambda_{8}+\left(k_{1}^{2}+k_{2}^{2}+n^{2}\right) \lambda_{9}\right. \\
& \left.+\left(k_{1}^{2}+k_{2}^{2}\right) \lambda_{10}+\left(v_{L}^{2}+v_{R}^{2}+\Lambda_{R}^{2}\right) \lambda_{12}+v_{L}^{2}\left(1+\frac{\Lambda_{R}^{2}}{v_{L}^{2}-v_{R}^{2}}\right) \lambda_{13}\right], \\
\mu_{\chi}^{2}= & -\left(v_{L}^{2}+v_{R}^{2}+\frac{v_{R}^{2} \Lambda_{R}^{2}}{v_{R}^{2}-v_{L}^{2}}\right) \lambda_{1}-\frac{1}{2}\left[\frac{\Lambda_{R}^{2} v_{L}^{2}}{\left(v_{L}^{2}-v_{R}^{2}\right)} \lambda_{2}+\left(k_{1}^{2}+k_{2}^{2}+n^{2}\right) \lambda_{7}+k_{1}^{2} \lambda_{8}+k_{3}^{2} \lambda_{12}\right. \\
& \left.+\frac{k_{3}^{2} v_{L}^{2}}{\left(v_{L}^{2}-v_{R}^{2}\right)} \lambda_{13}\right], \\
f_{\Phi}= & \frac{2\left(n^{2}-k_{2}^{2}\right) n k_{2} \lambda_{4}-n k_{2} k_{3}^{2} \lambda_{10}+k_{3} \Lambda_{R}\left(n^{2}-k_{2}^{2}\right) \lambda_{14}+n k_{2} \Lambda_{R}^{2} \lambda_{8}}{\sqrt{2} k_{1}\left(v_{L}^{2}-v_{R}^{2}\right)}, \\
f_{\rho}= & \frac{1}{\sqrt{2} k_{1}}\left\{n k_{2} \lambda_{14}+\frac{\Lambda_{R}\left(k_{1}^{2}-n^{2}\right)}{k_{3}} \lambda_{8}+\frac{v_{L}^{2} \Lambda_{R}}{k_{3}\left(v_{L}^{2}-v_{R}^{2}\right)}\left[k_{3}^{2} \lambda_{13}+\left(v_{L}^{2}-v_{R}^{2}-\Lambda_{R}^{2}\right)\left(2 \lambda_{1}-\lambda_{2}\right)\right]\right\}, \\
f_{\chi}= & \frac{v_{L} v_{R}\left[\left(2 \lambda_{1}-\lambda_{2}\right)\left(v_{R}^{2}+\Lambda_{R}^{2}-v_{L}^{2}\right)-k_{3}^{2} \lambda_{13}\right]}{\sqrt{2}\left(v_{R}^{2}-v_{L}^{2}\right) k_{1}},
\end{aligned}
$$

together with the relations

$$
\begin{aligned}
& 2 k_{1}^{2} k_{2} k_{3}^{2}\left(v_{L}^{2}-v_{R}^{2}\right) \lambda_{11}-n v_{L} v_{R} \Lambda_{R}^{2}\left[k_{3}^{2} \lambda_{13}+\left(2 \lambda_{1}-\lambda_{2}\right)\left(v_{L}^{2}-v_{R}^{2}-\Lambda_{R}^{2}\right)\right]=0 \\
& n k_{3}\left(k_{1}^{2}-k_{2}^{2}\right) \lambda_{14}+\left(n^{2}-k_{1}^{2}\right) k_{2} \Lambda_{R} \lambda_{8}+\frac{k_{3}^{2} v_{L}\left(n v_{R}-k_{2} v_{L}\right) \Lambda_{R}}{v_{L}^{2}-v_{R}^{2}} \lambda_{13} \\
& \quad+\frac{v_{L}\left(k_{2} v_{L}-n v_{R}\right) \Lambda_{R}\left(v_{L}^{2}-v_{R}^{2}-\Lambda_{R}^{2}\right)}{v_{L}^{2}-v_{R}^{2}}\left(\lambda_{2}-2 \lambda_{1}\right)=0, \\
& \left(k_{1}^{2}-k_{2}^{2}\right)\left[\left(k_{1}^{2}-n^{2}\right) \lambda_{4}+\frac{n^{2} k_{3}^{2}}{\left(n^{2}-k_{2}^{2}\right)} \lambda_{10}\right]+\frac{\lambda_{8}}{2}\left\{k_{1}^{2}\left(v_{L}^{2}+v_{R}^{2}\right)-\frac{\left[n^{4}+\left(k_{1}^{2}-2 n^{2}\right) k_{2}^{2}\right] \Lambda_{R}^{2}}{n^{2}-k_{2}^{2}}\right\} \\
& \quad+n k_{2} k_{3} \Lambda_{R} \lambda_{14}+\frac{v_{R}^{2}+\Lambda_{R}^{2}}{2\left(v_{L}^{2}-v_{R}^{2}\right)}\left[k_{3}^{2} v_{L}^{2} \lambda_{13}+v_{L}^{2}\left(v_{L}^{2}-v_{R}^{2}-\Lambda_{R}^{2}\right)\left(2 \lambda_{1}-\lambda_{2}\right)\right]=0 .
\end{aligned}
$$

Different patterns of symmetry breaking associated to different hierarchies of the above vacuum expectation values will thus translate into a different nature for the physics beyond the Standard Model expected above current LHC energies. In one of them the "next" theory will be left-right symmetric, while in the other it will follow the pattern dictated by the 331 gauge dynamics. 


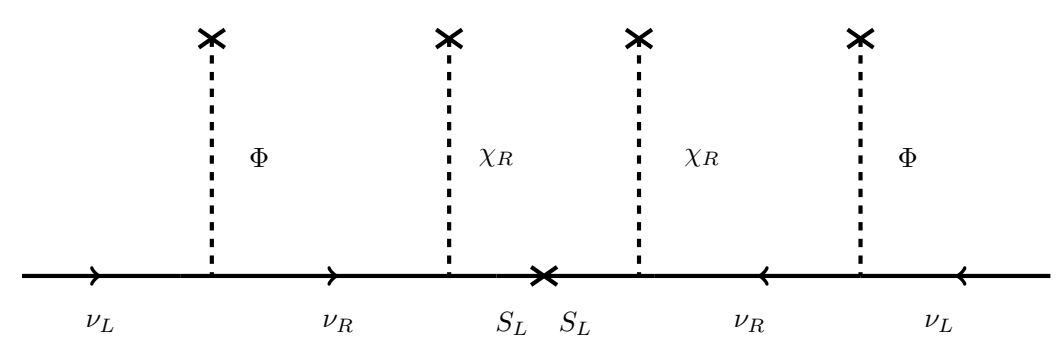

Figure 1. Inverse seesaw diagram.

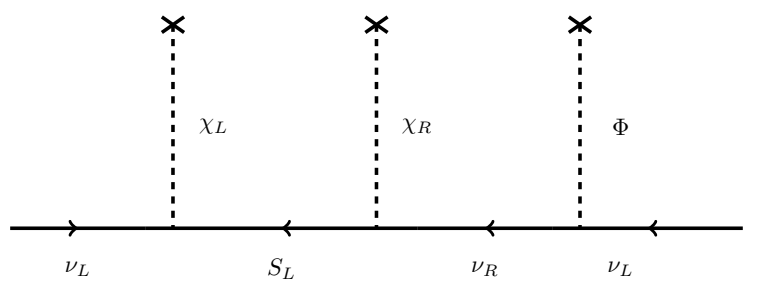

Figure 2. Linear seesaw diagram, up to symmetrization.

\section{Phenomenological implications}

After spontaneous symmetry breaking driven by the pattern in eq. (2.4) the neutrino mass matrix in the $\left(\nu_{L}, \nu_{R}^{c}, N_{L}, N_{R}^{c}, S_{L}\right)$ basis is given by:

$$
M_{\nu}=\left(\begin{array}{ccccc}
0 & y k_{1} & 0 & 0 & y_{L} v_{L} \\
y^{T} k_{1} & 0 & 0 & 0 & y_{R} v_{R} \\
0 & 0 & 0 & y n & 0 \\
0 & 0 & y^{T} n & 0 & y_{R} \Lambda_{R} \\
y_{L}^{T} v_{L} & y_{R}^{T} v_{R} & 0 & y_{R}^{T} \Lambda_{R} & \mu
\end{array}\right) .
$$

For example, setting $v_{L} \rightarrow 0$ gives us

$$
m_{\text {light }} \approx \frac{k_{1}^{2}}{v_{R}^{2}}\left(y y_{R}^{T-1}\right) \mu\left(y_{R}^{-1} y^{T}\right),
$$

which vanishes as $\mu \rightarrow 0$, reproducing the standard inverse seesaw relation $[10,11]$ illustrated in figure 1 . On the other hand, taking $\mu \rightarrow 0$ leads to

$$
m_{\mathrm{light}} \approx \frac{v_{L} k_{1}}{v_{R}}\left[y\left(y_{R} y_{L}^{-1}\right)^{T}+\left(y_{R} y_{L}^{-1}\right) y^{T}\right],
$$

recovering the linear seesaw mass relation [14], see figure 2. Besides, as dictated by parity, the Yukawa couplings satisfy $y_{L}=y_{R}$ and the above mass relation reduces to $m_{\text {light }} \approx \frac{v_{L} k_{1}}{v_{R}}\left(y+y^{T}\right)$. In either case we can naturally explain the lightness of neutrinos as a result of the exchange of quasi-Dirac heavy lepton messengers that could lie in the TeV scale $[24,25]$. The required mixing parameters measured in neutrino oscillation experiments can also be accommodated. 
In the quark sector, the up- and down-type quark mass matrices are determined by the Yukawa interactions

$$
-\mathcal{L}_{\text {quarks }}=\sum_{\alpha, \beta=1}^{2}\left(h_{\alpha \beta}^{Q} \bar{Q}_{L}^{\alpha} \Phi^{*} Q_{R}^{\beta}\right)+h_{33}^{Q} \bar{Q}_{L}^{3} \Phi Q_{R}^{3}+\sum_{\alpha=1}^{2}\left(h_{\alpha 3}^{Q} \bar{Q}_{L}^{\alpha} \rho^{*} Q_{R}^{3}+h_{3 \alpha}^{Q} \bar{Q}_{L}^{3} \rho Q_{R}^{\alpha}\right)+\text { h.c. }
$$

and are given by

$$
\begin{aligned}
& M^{u}=\frac{1}{\sqrt{2}}\left(\begin{array}{ccc}
h_{11}^{Q} k_{2} & h_{12}^{Q} k_{2} & 0 \\
h_{21}^{Q} k_{2} & h_{22}^{Q} k_{2} & 0 \\
-h_{31}^{Q} k_{3} & -h_{32}^{Q} k_{3} & h_{33}^{Q} k_{1}
\end{array}\right), \\
& M^{d}=\frac{1}{\sqrt{2}}\left(\begin{array}{ccc}
h_{11}^{Q} k_{1} & h_{12}^{Q} k_{1} & h_{13}^{Q} k_{3} \\
h_{21}^{Q} k_{1} & h_{22}^{Q} k_{1} & h_{23}^{Q} k_{3} \\
0 & 0 & h_{33}^{Q} k_{2}
\end{array}\right) .
\end{aligned}
$$

Concerning the neutral current weak interaction, these are exactly flavor-diagonal as expected by the Glashow-Iliopoulos-Maiani mechanism. There are, however, new neutral gauge bosons that couple non-diagonally to the quark flavors. Such flavor changing interactions are constrained by direct searches at the LHC Dilepton data, as well as by limits from K, D and B neutral meson systems [21]. On the other hand right-handed neutrino pair production through the $Z^{\prime}$ gauge boson portal would induce sizeable rates for lepton flavour violation effects at the high energies available at the LHC [25], even when processes like $\mu \rightarrow e \gamma$ are negligibly small.

\section{Flavor-changing neutral currents}

An interesting feature of our model is the break down of the Glashow-Iliopoulos-Maiani mechanism in the weak neutral current. Indeed there are flavor-changing neutral currents (FCNC) mediated by the new heavy neutral gauge bosons $Z_{L}^{\prime}$ and $Z_{R}^{\prime}$ associated to the left and right $\mathrm{SU}(3)$ groups. The neutral current Lagrangian is given by

$$
\begin{aligned}
\mathcal{L}_{\mathrm{NC}}= & -\bar{f}_{L i} \gamma^{\mu}\left[g_{L}\left(T_{3}^{L} W_{\mu}^{L 3}+T_{8}^{L} W_{\mu}^{L 8}\right)+g_{x} X_{i} B_{\mu}\right] f_{L i} \\
& -\bar{f}_{R i} \gamma^{\mu}\left[g_{L}\left(T_{3}^{R} W_{\mu}^{R 3}+T_{8}^{R} W_{\mu}^{R 8}\right)+g_{x} X_{i} B_{\mu}\right] f_{R i},
\end{aligned}
$$

with $X=Q-T_{L}^{3}-T_{R}^{3}+\frac{1}{\sqrt{3}}\left(T_{L}^{8}+T_{R}^{8}\right)$. We note that the Standard Model neutral gauge bosons, the photon and the standard $Z$ boson, do not mediate FCNC since their coupling involves only the electric charge and $T^{3}$ generators in flavor space, hence proportional to the identity matrix. However the $T_{8}$ piece of the neutral current leads to flavor change, and taking this into account we obtain the FCNC piece of the neutral current Lagrangian,

$$
\begin{aligned}
\mathcal{L}_{\mathrm{FCNC}} \supset & -\bar{f}_{L i} \gamma^{\mu} T_{8}^{L}\left(g_{L} W_{\mu}^{L 8}+\frac{1}{\sqrt{3}} g_{X} B_{\mu}\right) f_{L i} \\
& -\bar{f}_{R i} \gamma^{\mu} T_{8}^{R}\left(g_{L} W_{\mu}^{R 8}+\frac{1}{\sqrt{3}} g_{X} B_{\mu}\right) f_{R i} .
\end{aligned}
$$


Defining the relevant fields $Z_{L, R \mu}^{\prime}$ and couplings $g_{L, R}^{\prime}$ as

$$
\begin{aligned}
& g_{L}^{\prime} Z_{L \mu}^{\prime}=g_{L} W_{\mu}^{L 8}+\frac{1}{\sqrt{3}} g_{X} B_{\mu}, \\
& g_{R}^{\prime} Z_{R \mu}^{\prime}=g_{L} W_{\mu}^{R 8}+\frac{1}{\sqrt{3}} g_{X} B_{\mu},
\end{aligned}
$$

we can write down the FCNC Lagrangian

$$
\mathcal{L}_{\mathrm{FCNC}}=-\frac{1}{\sqrt{3}}\left[g_{L}^{\prime} Z_{L \mu}^{\prime}\left(V_{3 j}^{q L}\right)^{*} V_{3 i}^{q L} \overline{q_{L}^{\prime j}} \gamma^{\mu} q_{L}^{i}+g_{R}^{\prime} Z_{R \mu}^{\prime}\left(V_{3 j}^{q R}\right)^{*} V_{3 i}^{q R} \overline{q_{R}^{\prime j}} \gamma^{\mu} q_{R}^{\prime i}\right],
$$

with $q^{\prime}=(u, c, t)$ or $q^{\prime}=(d, s, b)$ and $i \neq j$. Thus, the effective Lagrangian for meson mixing becomes

$$
\mathcal{L}_{\mathrm{FCNC}}^{\mathrm{eff}}=\frac{1}{3}\left|\left(V_{3 j}^{q L}\right)^{*} V_{3 i}^{q L}\right|^{2}\left(\frac{g_{L}^{\prime 2}}{m_{Z_{L}^{\prime}}^{2}}\right)\left(\overline{q_{L}^{\prime j}} \gamma^{\mu} q_{L}^{\prime i}\right)^{2}+\frac{1}{3}\left|\left(V_{3 j}^{q R}\right)^{*} V_{3 i}^{q R}\right|^{2}\left(\frac{g_{R}^{\prime 2}}{m_{Z_{R}^{\prime}}^{2}}\right)\left(\overline{q_{R}^{\prime j}} \gamma^{\mu} q_{R}^{\prime i}\right)^{2} .
$$

The phenomenology of the model is quite rich since the $Z_{L, R}^{\prime}$ mediate meson mixing and can also be searched directly at the LHC by Drell Yann production in the dilepton channel. One notices that the relative importance of the left and right terms is determined by the hierarchy of vacuum expectation values which characterizes the physics "just beyond" the Standard Model in our scheme. Indeed, these gauge boson mass parameters scale as $m_{Z_{L}^{\prime}} \sim n$ and $m_{Z_{R}^{\prime}} \sim \sqrt{n^{2}+v_{R}^{2}}$ so that for $n \gg v_{R}$ the intermediate scale physics is described by the $\mathrm{SU}(2)_{\mathrm{L}} \otimes \mathrm{SU}(2)_{\mathrm{R}} \otimes \mathrm{U}(1)$ gauge structure. ${ }^{3}$ In this left-right symmetric limit the FCNC will be suppressed by the high value of $v_{R}$. In contrast, for $n \ll v_{R}$ one has intermediate $\mathrm{SU}(3)_{\mathrm{c}} \otimes \mathrm{SU}(3)_{\mathrm{L}} \otimes \mathrm{U}(1)$ physics at "low" scale. In this case one recovers the situation considered in [21] with FCNC suppressed by the magnitude of the vacuum expectation value $n$. For the generic case of potentially comparable magnitudes for $n$ and $v_{R}$ one can generalize the analysis given in [21] so as to constrain them jointly, by combining restrictions from meson mixing with LHC bounds from direct search. These will restrict the symmetry breaking path from our original "mother" theory.

Before concluding this brief sketch, let us mention that, since all leptons are in the $\mathbf{3}^{*}$ representation, there are no tree level flavor changing effects mediated by $Z_{L}^{\prime}$ nor $Z_{R}^{\prime}$ in this sector and, as a result, lepton flavour violation is adequately suppressed. The same occurs for exotic (third component) quarks: since the up-type belong to the $\mathbf{3}$ and down-type transform as $\mathbf{3}^{*}$ they do not give rise to flavor change.

\section{Conclusion}

In summary, we have proposed a framework where the origin of small neutrino mass, spontaneous parity violation and the existence of three fermion families all have a common origin, with neutrinos getting mass from low-scale seesaw, such as the inverse or the linear

\footnotetext{
${ }^{3}$ In contrast to the most popular left-right symmetric model here the low-scale is consistent with neutrino mass generation.
} 
seesaw mechanism. The framework specifies dynamically whether the nature of the new physics to show up in the next run of the LHC will follow a left-right symmetric pattern, or a chiral $\mathrm{SU}(3)_{\mathrm{c}} \otimes \mathrm{SU}(3)_{\mathrm{L}} \otimes \mathrm{U}(1)$ symmetry profile, with quark flavor changing interactions associated to new $Z^{\prime}$ gauge bosons. These patterns follow from minimizing the Higgs potential for different hierarchies of the relevant vacuum expectation values. The theory reconciles the existence of parity at a fundamental level with the quantum consistency requirement of having precisely three families of fermions, in which quarks must be assigned in a non-sequential way with the third family different from the others. The existence of a $Z^{\prime}$ gauge boson would be probed in Dilepton studies, and also provide a production portal for the right-handed neutrinos responsible for generating neutrino mass via a genuine lowscale seesaw mechanism. These new $Z^{\prime}$ gauge bosons could also induce sub-weak flavor changing transitions in the $\mathrm{K}, \mathrm{D}$ and $\mathrm{B}$ neutral meson systems.

\section{Acknowledgments}

Work supported by Spanish grants FPA2014-58183-P, Multidark CSD2009-00064, SEV2014-0398 (MINECO) and PROMETEOII/2014/084 (Generalitat Valenciana). C.A.V-A. acknowledges support from CONACyT (Mexico), grant 274397. M.R. was supported by JAEINT-16-00831.

Open Access. This article is distributed under the terms of the Creative Commons Attribution License (CC-BY 4.0), which permits any use, distribution and reproduction in any medium, provided the original author(s) and source are credited.

\section{References}

[1] A.G. Dias, C.A. de S. Pires and P.S. Rodrigues da Silva, The Left-Right $\mathrm{SU}(3)_{L} \times \mathrm{SU}(3)_{R} \times \mathrm{U}(1)_{X}$ Model with Light, keV and Heavy Neutrinos, Phys. Rev. D 82 (2010) 035013 [arXiv: 1003.3260] [INSPIRE].

[2] M. Reig, J.W.F. Valle and C.A. Vaquera-Araujo, Unifying left-right symmetry and 331 electroweak theories, Phys. Lett. B 766 (2017) 35 [arXiv:1611.02066] [INSPIRE].

[3] S.L. Adler, Axial vector vertex in spinor electrodynamics, Phys. Rev. 177 (1969) 2426 [INSPIRE].

[4] J.S. Bell and R. Jackiw, A PCAC puzzle: $\pi^{0} \rightarrow \gamma \gamma$ in the $\sigma$-model, Nuovo Cim. A 60 (1969) 47 [INSPIRE].

[5] M. Singer, J.W.F. Valle and J. Schechter, Canonical Neutral Current Predictions From the Weak Electromagnetic Gauge Group SU(3) × U(1), Phys. Rev. D 22 (1980) 738 [inSPIRE].

[6] J.W.F. Valle and M. Singer, Lepton Number Violation With Quasi Dirac Neutrinos, Phys. Rev. D 28 (1983) 540 [INSPIRE].

[7] R.M. Fonseca and M. Hirsch, A flipped 331 model, JHEP 08 (2016) 003 [arXiv:1606.01109] [INSPIRE].

[8] F. Pisano and V. Pleitez, An SU(3) $\times \mathrm{U}(1)$ model for electroweak interactions, Phys. Rev. D 46 (1992) 410 [hep-ph/9206242] [INSPIRE]. 
[9] P.H. Frampton, Chiral dilepton model and the flavor question, Phys. Rev. Lett. 69 (1992) 2889 [INSPIRE].

[10] R.N. Mohapatra and J.W.F. Valle, Neutrino Mass and Baryon Number Nonconservation in Superstring Models, Phys. Rev. D 34 (1986) 1642 [InSPIRE].

[11] M.C. Gonzalez-Garcia and J.W.F. Valle, Fast Decaying Neutrinos and Observable Flavor Violation in a New Class of Majoron Models, Phys. Lett. B 216 (1989) 360 [InSPIRE].

[12] E.K. Akhmedov, M. Lindner, E. Schnapka and J.W.F. Valle, Left-right symmetry breaking in NJLS approach, Phys. Lett. B 368 (1996) 270 [hep-ph/9507275] [INSPIRE].

[13] E.K. Akhmedov, M. Lindner, E. Schnapka and J.W.F. Valle, Dynamical left-right symmetry breaking, Phys. Rev. D 53 (1996) 2752 [hep-ph/9509255] [InSPIRE].

[14] M. Malinsky, J.C. Romao and J.W.F. Valle, Novel supersymmetric $\mathrm{SO}(10)$ seesaw mechanism, Phys. Rev. Lett. 95 (2005) 161801 [hep-ph/0506296] [INSPIRE].

[15] M.E. Catano, R. Martinez and F. Ochoa, Neutrino masses in a 331 model with right-handed neutrinos without doubly charged Higgs bosons via inverse and double seesaw mechanisms, Phys. Rev. D 86 (2012) 073015 [arXiv:1206.1966] [InSPIRE].

[16] M. Gell-Mann, P. Ramond and R. Slansky, Complex Spinors and Unified Theories, Conf. Proc. C 790927 (1979) 315 [arXiv: 1306.4669] [InSPIRE].

[17] T. Yanagida, Horizontal symmetry and masses of neutrinos, Conf. Proc. C7902131 (1979) 95 [INSPIRE].

[18] R.N. Mohapatra and G. Senjanović, Neutrino mass and spontaneous parity violation, Phys. Rev. Lett. 44 (1980) 912 [INSPIRE].

[19] J. Schechter and J.W.F. Valle, Neutrino Masses in $\mathrm{SU}(2) \times \mathrm{U}(1)$ Theories, Phys. Rev. D 22 (1980) 2227 [INSPIRE].

[20] G. Lazarides, Q. Shafi and C. Wetterich, Proton Lifetime and Fermion Masses in an $\mathrm{SO}(10)$ Model, Nucl. Phys. B 181 (1981) 287 [INSPIRE].

[21] F.S. Queiroz, C. Siqueira and J.W.F. Valle, Constraining Flavor Changing Interactions from LHC Run-2 Dilepton Bounds with Vector Mediators, Phys. Lett. B 763 (2016) 269 [arXiv: 1608.07295] [INSPIRE].

[22] ATLAS collaboration, Search for resonances decaying to photon pairs in $3.2 \mathrm{fb}^{-1}$ of $\mathrm{pp}$ collisions at $\sqrt{s}=13 \mathrm{TeV}$ with the ATLAS detector, ATLAS-CONF-2015-081 (2015).

[23] D.T. Huong and P.V. Dong, Left-right asymmetry and $750 \mathrm{GeV}$ diphoton excess, Phys. Rev. D 93 (2016) 095019 [arXiv: 1603.05146] [INSPIRE].

[24] M. Dittmar, A. Santamaria, M.C. Gonzalez-Garcia and J.W.F. Valle, Production Mechanisms and Signatures of Isosinglet Neutral Heavy Leptons in $Z^{0}$ Decays, Nucl. Phys. B 332 (1990) 1 [INSPIRE].

[25] F.F. Deppisch, N. Desai and J.W.F. Valle, Is charged lepton flavor violation a high energy phenomenon?, Phys. Rev. D 89 (2014) 051302 [arXiv: 1308.6789] [INSPIRE]. 